\title{
Contribution to the ongoing discussion on fluoride toxicity
}

\author{
Sabine Guth ${ }^{1}$ (D) Stephanie Hüser ${ }^{1} \cdot$ Angelika Roth $^{1} \cdot$ Gisela Degen $^{1} \cdot$ Patrick Diel $^{2} \cdot$ Karolina Edlund $^{1}$. \\ Gerhard Eisenbrand ${ }^{3} \cdot$ Karl-Heinz Engel $^{4} \cdot$ Bernd Epe $^{5} \cdot$ Tilman Grune $^{6} \cdot$ Volker Heinz $^{7} \cdot$ Thomas Henle $^{8}$. \\ Hans-Ulrich Humpf ${ }^{9} \cdot$ Henry Jäger $^{10} \cdot$ Hans-Georg Joost ${ }^{11}$. Sabine E. Kulling ${ }^{12}$. Alfonso Lampen ${ }^{13} \cdot$ Angela Mally $^{14}$. \\ Rosemarie Marchan $^{1} \cdot$ Doris Marko $^{15} \cdot$ Eva Mühle $^{1} \cdot$ Michael A. Nitsche $^{16,17} \cdot$ Elke Röhrdanz $^{18} \cdot$ Richard Stadler $^{19}$. \\ Christoph van Thriel ${ }^{1} \cdot$ Stefan Vieths ${ }^{20} \cdot$ Rudi F. Vogel $^{21} \cdot$ Edmund Wascher $^{22} \cdot$ Carsten Watzl $^{23} \cdot$ Ute Nöthlings $^{24}$. \\ Jan G. Hengstler ${ }^{1}$
}

Received: 17 February 2021 / Accepted: 4 May 2021 / Published online: 6 June 2021

(c) The Author(s) 2021

\begin{abstract}
Since the addition of fluoride to drinking water in the 1940s, there have been frequent and sometimes heated discussions regarding its benefits and risks. In a recently published review, we addressed the question if current exposure levels in Europe represent a risk to human health. This review was discussed in an editorial asking why we did not calculate benchmark doses (BMD) of fluoride neurotoxicity for humans. Here, we address the question, why it is problematic to calculate BMDs based on the currently available data. Briefly, the conclusions of the available studies are not homogeneous, reporting negative as well as positive results; moreover, the positive studies lack control of confounding factors such as the influence of wellknown neurotoxicants. We also discuss the limitations of several further epidemiological studies that did not meet the inclusion criteria of our review. Finally, it is important to not only focus on epidemiological studies. Rather, risk analysis should consider all available data, including epidemiological, animal, as well as in vitro studies. Despite remaining uncertainties, the totality of evidence does not support the notion that fluoride should be considered a human developmental neurotoxicant at current exposure levels in European countries.
\end{abstract}

\section{Introduction}

Since the 1940s, fluoride has been added to drinking water in many countries as a means of caries prophylaxis. Fluoride prevents caries at low exposure levels, whereas, excessive fluoride exposure causes dental and skeletal fluorosis in humans, and developmental toxicity in animals. Based on this background, the European Food Safety Authority (EFSA) defined an adequate intake (AI) level for fluoride of $50 \mu \mathrm{g} / \mathrm{kg}$ b.w. at which the caries preventive effect approached its maximum whilst the risk of dental fluorosis

Sabine Guth, Stephanie Hüser and Angelika Roth contributed equally to this publication.

Ute Nöthlings and Jan G. Hengstler are co-senior authors.

Ute Nöthlings

noethlings@uni-bonn.de

$\triangle$ Jan G. Hengstler

hengstler@ifado.deAuthors and Affiliations

Extended author information available on the last page of the article approached its minimum (EFSA 2013). In recent years, the benefits and risks of fluoride exposure to the general population, e.g. by drinking water, fluoridated salt or dental care products, have been heavily debated, and special focus is set on potential adverse health effects, such as neurodevelopmental toxicity.

\section{What type of data is needed to assess fluoride developmental neurotoxicity?}

To adequately address potential human health concerns caused by exposure to fluoride, the available evidence from all sources should be included. Thus, it is crucial to critically review the evidence from epidemiological, as well as from animal and in vitro studies. Recently, we published a comprehensive review considering the available data from all the study types mentioned above, particularly focusing on developmental toxicity (Guth et al. 2020). Another factor to consider when assessing the potential health risks of fluoride is the expected level of exposure. The focus of our review 
was on studies investigating the developmental effects of fluoride levels in drinking water in the range of community water fluoridation (CWF) of $0.7-1.0 \mathrm{mg} / \mathrm{L}$, as well as naturally occurring exposure scenarios in Europe which generally do not exceed the AI defined by EFSA. Since our aim was to evaluate whether fluoride exposure in European countries is of potential health concern, we did not address other exposure scenarios, e.g. in areas with endemically occurring high fluoride concentrations in ground and drinking water.

In comparison, other reviews evaluating a potential developmental toxicity of fluoride (e.g. Choi et al. 2012; Grandjean 2019; Grandjean and Landrigan 2014) (i) focused on the evidence from epidemiological studies, but did not include experimental evidence, and/or (ii) included results from endemically high fluoride areas. Thus, it is important to recognize that our review, in comparison to others recently published on fluoride toxicity, aimed to address different questions, and this is reflected by the application of different inclusion criteria used. It is therefore not surprising that conclusions drawn by the authors differ in some respects.

Below, evidence from animal, in vitro and epidemiological studies is briefly summarized primarily focusing on European exposure scenarios as discussed in our review by Guth et al. (2020).

\section{Evidence from animal studies}

Chronic toxicity studies in rats, mice, and rabbits that focused on systemic effects of fluoride resulted in Lowest-Observed-Adverse-Effect Levels (LOAELs) ranging between 4.3 and $7.6 \mathrm{mg} / \mathrm{kg}$ b.w./day fluoride, and noobserved-adverse-effect levels (NOAELs) between 2.5 and $7.6 \mathrm{mg} / \mathrm{kg}$ b.w./day fluoride. Four well-conducted developmental toxicity studies (Collins et al. 2001, 1995; Heindel et al. 1996) are available which are in accordance with standard guidelines, used adequate numbers of animals, and administered sodium fluoride in drinking water. These studies resulted in NOAELs of $8.5-13.7 \mathrm{mg} / \mathrm{kg}$ b.w./day fluoride for rats and rabbits. It should be noted that the influence of specific fluoride doses on plasma levels may vary between different species. For example, it has been suggested that approximately fivefold higher doses in drinking water might be required for rats to achieve serum concentrations similar to those in humans (Dunipace et al. 1995; NRC 2006). However, it must also be taken into account that numerous variables could influence these relationships in both animal and human studies and the factor to calculate plasma concentrations is largely uncertain, in part because it could change with age or duration of exposure (NRC 2006).

To our knowledge, there are currently no further developmental studies that were performed according to standard guidelines. A search of the literature published between
2005 and 2018 revealed a number of animal studies that reported an effect of fluoride exposure on various endpoints in offspring during development (see (Guth et al. 2020)). We reviewed the quality of these studies and identified various limitations (see Box 1) that hamper their interpretation, thus reducing their value for risk assessment.

Box 1 Limitations (criteria not fulfilled) observed in animal studies investigating developmental or neurobehavioral effects

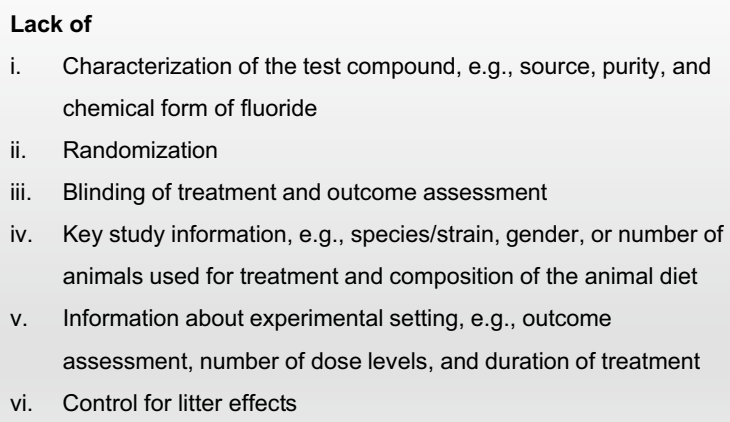

Studies investigating neurobehavioral toxicity in animals produced conflicting results (NTP 2016). A systematic review by the US National Toxicology Program (NTP) reported a low to moderate level of evidence of adverse effects on learning and memory in rats and mice exposed to fluoride concentrations substantially higher than $0.7 \mathrm{mg} / \mathrm{L}$ (NTP 2016). After the publication of the NTP report in 2016, several studies became available that investigated the impact of fluoride exposure on memory and learning in experimental animals. We reviewed the quality of the available studies and found that only two fulfilled the criteria listed in Box 1, but nevertheless still had limitations (McPherson et al. 2018; Pulungan et al. 2018). In both studies, no exposure-related differences in motor, sensory, or learning and memory performance were observed with the exposure levels investigated (up to $20 \mathrm{mg}$ fluoride/L or $9 \mathrm{mg}$ fluoride/ $\mathrm{kg}$ b.w./day, respectively). Of note, one study (McPherson et al. 2018) primarily investigated the influence of fluoride exposure during development on neurobehavioral aspects. The other 11 studies identified in our search had various and strong limitations, and did not meet key quality criteria discussed in detail in our review (see (Guth et al. 2020)).

\section{Human exposure in relation to adverse effects in animal experiments}

The mean intake of fluoride from water, food, beverages and oral hygiene products in European populations is usually below the AI recommended by EFSA. Recently, there has 
been some debate as to whether exposure in the range of the AI, i.e. $50 \mu$ g fluoride $/ \mathrm{kg}$ b.w. /day, is sufficient to cause an increased risk of adverse effects in humans. It has also been suggested that fluoride, at current exposure levels, should be categorized as a human developmental neurotoxicant, and be placed in the same category as lead, methyl mercury, arsenic and polychlorinated biphenyls (Grandjean 2019; Grandjean and Landrigan 2014). To evaluate the situation, we calculated a margin of exposure (MoE) between doses showing no adverse effects in animal studies and the AI (Guth et al. 2020). The lowest NOAEL for systemic toxicity from a welldesigned chronic animal study was $2.5 \mathrm{mg} / \mathrm{kg}$ b.w./day. The lowest NOAEL for developmental toxicity was $8.5 \mathrm{mg} / \mathrm{kg}$ b.w./day. Compared to the AI of $50 \mu \mathrm{g} / \mathrm{kg} / \mathrm{day}$, the margin of exposure (MoE) is $\sim 50$ (systemic toxicity) or $\sim 170$ (developmental toxicity), which are high MoEs.

\section{Evidence from in vitro studies}

Recent findings suggest that in vitro data should also be considered in the risk evaluation of chemicals (Godoy et al. 2013; Leist 2017). Therefore, we compared the highest reported fluoride concentrations in plasma of healthy individuals ( $3 \mu \mathrm{M}$; summarized by Guth et al. 2020; e.g. Rugg-Gunn et al. 2011) to cell culture medium concentrations causing cytotoxic effects in neuronal and stem cells of rodent and human origin, which occurred at $\sim 1 \mathrm{mM}$ in most studies (range: 0.1-4 mM) (Guth et al. 2020). This results in a ratio of $\sim 300$, which demonstrates that human plasma concentrations of fluoride are far below cytotoxic levels.

\section{Evidence from epidemiological studies}

Since our review (Guth et al. 2020) addressed the exposure scenarios relevant for European countries, we focused on epidemiological studies conducted in non-endemic fluoride areas or areas with CWF. Furthermore, we based our assessment on prospective studies in which cohorts were followed over a period of time (see inclusion criteria, Box 3). Two prospective cohort studies conducted in CWF areas that considered possible confounding factors (Broadbent et al. 2015; Green et al. 2019, Box 5) were included in our evaluation, and both reported conflicting results. In our review, we also noted that the majority of epidemiological studies conducted in areas with endemically occurring high fluoride levels in ground and drinking water reported an association between lower measures of intelligence and high fluoride exposure.

Other reviews (e.g. Choi et al. 2012; Grandjean 2019; Grandjean and Landrigan 2014) did not only focus on community water fluoridation and prospective cohort studies, but also included cross-sectional epidemiological studies, as well as studies performed in areas with endemically occurring high fluoride concentrations in drinking water. In these reviews, it was concluded that recent epidemiological evidence suggests that elevated fluoride intake during early development can result in considerable IQ deficits (Grandjean 2019).

While the present letter was under review, an article on a retrospective cohort study was published performed by a research institute under the Swedish Ministry of Employment (Institute for Evaluation of Labour Market and Education Policy; IFAU), which estimated a zero effect on cognitive ability for fluoride levels in Swedish drinking water (Aggeborn and Oehman 2021). This article is based on data of a comprehensive retrospective cohort study already discussed in our previous review (Aggeborn and Oehman 2017; see also Guth et al. 2020).

\section{Limitations of epidemiological studies and inclusion criteria}

Our analysis of the epidemiological studies repeatedly identified the limitations summarized in Box 2. In line with our goal to assess possible effects of fluoride at current exposure levels in Europe, we used the inclusion criteria summarized in Box 3. However, in a recent editorial (Spittle 2020), the author wrote that we omitted specific studies in the epidemiological section of our manuscript (Guth et al. 2020). This was indeed the case, because these studies did not meet our inclusion criteria. We provide a standardized profile and brief discussion of these studies with their strength and limitations, while simultaneously addressing the comments of Spittle (2020) (Box 4). These studies do not change the overall conclusion that the totality of currently available scientific evidence does not support the concept that fluoride should be assessed as a human developmental neurotoxicant at the current exposure levels in Europe. Furthermore, the authors of the studies (Box 4) were aware of these limitations and usually addressed them in the corresponding discussions. 
Box 2 Limitations observed in previously published epidemiological studies about fluoride exposure and intelligence (IQ)

- Studies were often performed in relatively poor rural regions with unusually high concentrations of fluoride in drinking water, and were compared to 'reference populations' with fluoride drinking water concentrations in the recommended range. It should be considered that unusual fluoride concentrations in drinking water may be associated with a less developed health-care system and lower socioeconomic status.

- Studies were performed in fluoride endemic areas and did not consider the influence of other neurotoxicants, such as arsenic (and other contaminants) in groundwater.

- The IQ of the parents was not considered in some studies. The 'outcome' (intelligence) may influence the 'input' (fluoride exposure).

Explanation: It is possible that parents with higher IQ read or inform themselves about the possible health hazards to children, and therefore avoid fluoride exposure. In this case high maternal/parental intelligence would be causally linked to lower fluoride exposure rather than high fluoride exposure causing lower intelligence in children.

- $\quad \mathrm{IQ}$ analysis was not optimally performed to allow a reliable evaluation of intelligence.

Example: IQ was analyzed only once between the ages of 3 and 4 when the $I Q$ of children is known to change rapidly, but the exact age was not considered.

- Statistical significance was reported, although removing only one or two cases with extreme IQ scores from the models would result in non-significant associations.

- In some studies, which were based on previous larger programs, the authors did not describe why only a fraction of the individuals was chosen for the particular study.

Example: In one study, only 610 of 2001 pregnant women from the MIREC program were considered and information on maternal urinary fluoride was missing in a relatively high fraction of the mothers whose children's $I Q$ was determined. This may represent a source of bias. 


\section{Box 3 Inclusion criteria of Guth et al. (2020): Epidemiological studies used for risk assessment}

- Publication period: Published between January 2012 and August 2019 (Pubmed search)

- Study type: Prospective cohort study

Explanation: Cross-sectional and ecological studies do not allow for the establishment of causal relationships and are not appropriate to ultimately evaluate the effect of chronic fluoride exposure on a parameter like human intelligence, but serve to derive hypotheses. In contrast, prospective studies in which cohorts are followed over a period of time, and data relating to predetermined exposures and outcomes that are collected over time, are considered appropriate for inferring causality. Thus, results from cross-sectional studies were discussed in the review, but not included in risk assessment.

- Endpoint: Availability of the intelligence quotient (IQ)

Explanation: Since IQ is a widely accepted, reliable and comparable endpoint, we included only epidemiological and ecological studies where both IQ and fluoride exposure were available. Studies relying on other endpoints e.g. cognitive function, mental and psychomotor development were excluded.

- Study region: We focused on studies with community water fluoridation (CWF); studies in areas with endemically-occurring high fluoride were not included.

Explanation: Almost all studies investigating the effect of fluoride intake on intelligence were performed in relatively poor, rural communities, e.g. in China, Iran, Mexico and Mongolia. In these areas, drinking water may contain comparatively high levels of fluoride ('exposed population'); whereas, the 'reference populations' often had access to water containing fluoride at the recommended level (critically discussed by Feldman 2014; Gelinas and Allukian 2014). This constellation may lead to a confounding effect; rural regions with unusually high or unusually low fluoride in drinking water may be associated with a less developed health-care system, as well as lower educational and socioeconomic status. Furthermore, in these regions the overall nutritional status and the intake of essential nutrients may be lower and the exposure to environmental contaminants such as lead, cadmium, mercury, or manganese may be higher-factors that are also discussed as having a potential impact on intelligence. Furthermore, to maintain electroneutrality, (drinking) water with higher concentrations of endemically occurring fluoride must contain higher concentrations of positive ions to balance out the fluoride. This may influence the $\mathrm{pH}$ of the water or result in greater contamination by electropositive water contaminants, for example aluminum, zinc, arsenic, lead, mercury, and other metals and metalloids. Thus, in studies on naturally-occurring fluoride, it is important to control for these contaminants. In contrast, in studies on community water fluoridation the negative fluoride ions are balanced out in the water treatment process; therefore, other substances are unlikely to be a source of confounding. Thus, results from areas with CWF are more reliable, which has prompted us to focus our risk assessment on CWF studies. 
Box 4 Standardized profiles of the individual epidemiological studies mentioned in the editorial of Spittle (2020)

\section{Bashash et al. 2017}

\section{Key message}

Higher prenatal fluoride exposure, in the general range of exposures reported for other general population samples of pregnant women and non-pregnant adults, was associated with lower scores on tests of cognitive function in the offspring at age 4 and $6-12$ years.

\begin{tabular}{|c|c|}
\hline $\begin{array}{l}\text { - } \text { Study type: } \mathrm{P} \\
\text { - } \text { Study region: } \\
\text { - } \quad \text { Investigated }\end{array}$ & $\begin{array}{l}\text { ective } \\
\text { emically occurring fluoride } \\
\text { oint: IQ }\end{array}$ \\
\hline Strength & Limitations \\
\hline $\begin{array}{l}\text { - Prospective study design } \\
\text { - Individual exposure assessment (urinary fluoride } \\
\text { concentrations) } \\
\text { - Urinary fluoride concentrations were corrected for dilution } \\
\text { (creatinine or specific gravity) } \\
\text { - Data on maternal IQ were available } \\
\text { - Consideration of numerous covariates (adjustment for } \\
\text { gestational age, weight at birth, sex, parity (being the first } \\
\text { child), age at outcome measurement, and maternal } \\
\text { characteristics including smoking history (ever smoked vs. } \\
\text { nonsmoker), marital status (married vs. others), age at } \\
\text { delivery, IQ, education, socioeconomic status, cohort and } \\
\text { exposure to other neurotoxicants such as lead and mercury) } \\
\text { - } 6 \text { - } 12 \text { years of follow-up }\end{array}$ & $\begin{array}{l}\text { - Lack of data on fluoride content in water } \\
\text { - A relatively large part of the participants (around } 40 \% \text { ) } \\
\text { provided only one spot urine sample } \\
\text { - Conducted in an endemic fluoride area, but the impact of } \\
\text { known potential neurotoxins occurring in the study region, } \\
\text { e.g. arsenic, manganese, PCBs was not considered } \\
\text { - Covariates not considered: fluoride exposure via diet, iodine } \\
\text { in salt, alcohol consumption of the mothers, breastfeeding, } \\
\text { maternal exposure to the neurotoxicant arsenic }\end{array}$ \\
\hline
\end{tabular}

Explanation: Participants from the Early Life Exposures in Mexico to Environmental Toxicants (ELEMENT) project were studied. Fluoride was measured in archived spot urine samples taken from mothers during pregnancy and from their children when 6-12 y old, adjusted for urinary creatinine and specific gravity, respectively. Intelligence of children was measured by the General Cognitive Index $(\mathrm{GCl})$ of the McCarthy Scales of Children's Abilities at age 4 and full-scale intelligence quotient (IQ) from the Wechsler Abbreviated Scale of Intelligence (WASI) at age 6-12. Higher prenatal exposure to fluoride in the general range of exposures reported for other general population samples of pregnant women and non-pregnant adults, was associated with lower $\mathrm{GCl}$ Scores and with lower FullScale IQ scores. The authors noted as a limitation of the study that fluoride was measured in spot (second morning void) urine samples instead of 24-hr urine collections. However, it was also emphasized that a close relationship between the fluoride concentrations of early morning samples and 24-hr specimens have been reported. Other limitations mentioned by the authors include the lack of information about iodine in salt, which could have an influence on cognition; the lack of data on fluoride content in the water consumed; and the lack of information on exposure to the neurotoxicant arsenic.

Of note, the ELEMENT birth cohort was conducted to investigate early life exposures to environmental toxicants in Mexico City. Relatively high concentrations of numerous contaminants were detected in urinary and/or blood samples of pregnant women and their children. For example, potential developmental neurotoxicants such as manganese (Claus Henn et al. 2018), and various other metals (arsenic, lead, cadmium, aluminium etc. (Lewis et al. 2018)), or biomarkers of phyrethroid exposure (3-phenoxybenzoic acid) (Watkins et al. 2016) were found to be present in urine or other biological samples. Furthermore, phthalate metabolites and bisphenol $A$ were detected in maternal urinary samples (Watkins et al. 2017). The finding, that inhabitants of Mexico City might be exposed to various other neurotoxicants is further supported by results from other cohort studies conducted in Mexico City, e.g. the PROGRESS cohort (Programming Research in Obesity, Growth, Environment and Social Stressors) that determined an exposure to arsenic (Mullin et al. 2019) or manganese (Kupsco et al. 2019) from investigation of maternal blood samples, or another cohort from Mexico City that detected concentrations of potentially neurotoxic persistent organic pollutants e.g. polychlorinated biphenyls (PCBs) in blood samples (Orta-Garcia et al. 2014).

Even though the study of Bashash et al. is a prospective, longitudinal study and is therefore of suitable study design, it was conducted in a fluoride endemic area and showed limitations concerning an adequate consideration of exposure to known neurotoxicants. Therefore, the study was not included in our evaluation focusing on fluoride exposure levels in the range of CWF or European exposure scenarios (Guth et al. 2020). 


\section{Valdez-Jimenez et al. 2017}

\section{Key message}

Mental development (MDI) showed an inverse association with fluoride levels in maternal urine during pregnancy suggesting that cognitive alterations in children born from fluoride exposed mothers could start in early prenatal stages of life.

\begin{tabular}{|c|c|}
\hline 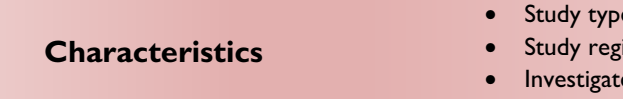 & $\begin{array}{l}\text { ive } \\
\text { ically occurring high fluoride } \\
\text { t: Mental and Psychomotor Development (MDI and PDI) }\end{array}$ \\
\hline Strength & Limitations \\
\hline $\begin{array}{l}\text { - Prospective study design } \\
\text { - Individual exposure assessment (individual water samples, } \\
\text { urinary fluoride concentrations) }\end{array}$ & $\begin{array}{l}\text { - Small sample size } \\
\text { - Short period of follow-up } \\
\text { - Only a small percentage of the participants provided biological } \\
\text { samples } \\
\text { - Maternal IQ was not reported } \\
\text { - Conducted in an endemic fluoride area, but the impact by other } \\
\text { neurotoxicants (lead, mercury, arsenic) was not considered } \\
\text { - Small set of covariates and sensitivity variables was used } \\
\text { (gestational age, type of water for consumption, marginalization } \\
\text { index and age of child) } \\
\text { - Urinary fluoride concentrations were not corrected for } \\
\text { creatinine }\end{array}$ \\
\hline
\end{tabular}

Explanation: The association between in utero exposure to fluoride and Mental and Psychomotor Development (MDI and PDI) was evaluated through the Bayley Scale of Infant Development II (BSID-II) in infants at age 3-I5 month (average of 8 month). The sample included 65 mother-infant pairs from two endemic hydrofluorosis areas in Mexico (Durango City and Lagos de Moreno, Jalisco, Mexico). Environmental exposure to fluoride was quantified in tap and bottled water samples and in maternal urine; samples were collected during the Ist, 2nd and 3rd trimester of pregnancy. The mean values of fluoride in tap water for the Ist, 2 nd and 3rd trimester were between 2.6 and $3.7 \mathrm{mg} / \mathrm{L}$; more than $80 \%$ of the samples exceeded the reference value of $1.5 \mathrm{mg} / \mathrm{L}$ with a maximum value of $12.5 \mathrm{mg} / \mathrm{L}$. Maternal urinary fluoride levels were found to be associated with statistically significantly lower MDI scores after adjusting for gestational age, age of child, a marginality index, and type of drinking water.

As also noted by the authors this study has some limitations including the small sample size of evaluated children, the short period of follow-up and the low participation to provide biological and environmental samples in the last trimester. It was conducted in residents from endemic fluoride areas and the impact by other neurotoxicants e.g. of mercury, arsenic, and lead was not considered. Furthermore, only a small set of covariates and sensitivity variables was used (gestational age, age of child, marginalization index and type of water for consumption) and urinary fluoride measures were not corrected for creatinine (which, by adjusting for urinary dilution effects, provides a more reliable measure of internal fluoride exposure). It should also be noted that although BSID-I and -II are validated as end points for primary neurodevelopment measures and can be used to assess early developmental delays, both are not designed to predict long-term neurocognitive outcome (Sun et al. 2015). Furthermore, BSID-I and -II do not have adequate measures to eliminate the confounding source resulting from the cultural influence (Sun et al. 2015). It is well known that during the assessment procedure the responses can be significantly altered depending on the individual cultural background and/or ethnicity (Sun et al. 2015). 


\section{Xiang et al. 2003}

\section{Key message}

In endemic fluorosis areas, drinking water fluoride levels greater than $1.0 \mathrm{mg} / \mathrm{L}$ may adversely affect the development of children's intelligence.

\section{Characteristics}

- Study type: cross-sectional

- Study region: endemically occurring high fluoride

- Investigated endpoint: IQ

\begin{tabular}{|c||l|}
\hline Strength & Limitations \\
\hline \begin{tabular}{|l|l|}
\hline - Individual exposure assessment (water fluoride \\
concentrations; in part urinary fluoride concentrations)
\end{tabular} & $\begin{array}{l}\text { Cross-sectional study design } \\
\text { Conducted in an endemic fluoride area, but the impact by } \\
\text { other neurotoxicants (lead, mercury, arsenic) was not } \\
\text { considered } \\
\text { Small set of covariates and sensitivity variables was used } \\
\text { (urinary iodine, family income, or parent's education } \\
\text { level) } \\
\text { Maternal IQ was not reported } \\
\text { Urinary fluoride measures were not available for all } \\
\text { participants }\end{array}$ \\
\hline
\end{tabular}

Explanation: The study by Xiang et al. (2003) was already evaluated by NRC in 2006. NRC summarized that several studies from China have reported the effects of fluoride in drinking water on cognitive capacities (NRC, 2006). In the opinion of the NRC, among the studies, the one by Xiang et al. (2003) had the strongest design. However, it was noted that overall the significance of these Chinese studies is uncertain (NRC, 2006).

The study by Xiang et al. has a cross sectional study design which cannot be used to determine causality. The Intelligence Quotient (IQ) was measured in 512 children, aged 8-13 years, living in two villages in China, differing in the level of fluoride in their drinking water. Xiang et al. analyzed individual water fluoride levels; however, not all participants provided urinary samples ( $47 \%$ in the village Xinhuai; $70 \%$ in the village Wamiao). In the high-fluoride village of Wamiao the mean water fluoride level was reported at $2.47 \pm 0.79 \mathrm{mg} / \mathrm{L}$ ( $\mathrm{range}$ $0.57-4.50 \mathrm{mg} / \mathrm{L}$ ). In the low-fluoride village of Xinhuai the mean water fluoride level was reported at $0.36 \pm 0.15 \mathrm{mg} / \mathrm{L}$ (range: $0.18-0.76$ $\mathrm{mg} / \mathrm{L})$.

The fluoride levels in drinking water of the high-fluoride village Wamiao were able to be stratified into five groups. The rates of mental retardation (IQ <70) and borderline intelligence (IQ 70-79) of the two groups with the lowest water fluoride levels of the high-fluoride village of Wamiao $(0.75$ and $1.53 \mathrm{mg} / \mathrm{L}$ fluoride, mean) did not differ significantly from that of children from the low-fluoride village Xinhuai. A significant effect on mental retardation compared to the Xinhuai group was observed only at mean fluoride concentrations of $2.46 \mathrm{mg} / \mathrm{L}$ fluoride and higher $(\mathrm{p}<0.0 \mathrm{I})$. The mean IQ of children in Wamiao did not differ significantly from the mean IQ of children in Xinhuai in the $0.75 \mathrm{mg} / \mathrm{L}$ fluoride group, but was statistically different in the $1.53 \mathrm{mg} / \mathrm{L}$ fluoride group $(p<0.05)$ and also in groups with a mean fluoride concentration of $2.46 \mathrm{mg} / \mathrm{L}$ fluoride and higher $(\mathrm{p}<0.01)$. The children's IQs were not related to urinary iodine, family income, or parent's education level. Furthermore, the authors of the study determined a reference value concentration of $0.925 \mathrm{mg}$ fluoride/L and stated that this is very close to the current national fluoride standard for China of $<1.0 \mathrm{mg} F / L$ suggesting that the current national standard is safe enough to protect $90 \%$ of children, aged 8-13 years, from adverse effects on their intelligence development. This reference value also reflects the range of CWF in several countries or is even somewhat higher. 
Soto-Barreras et al. 2019

\section{Key message}

The study results suggest that exposure to fluoride reduces the prevalence of dental caries, but no association was found to the intelligence of children.

\begin{tabular}{|l|l|}
\hline & - Study type: cross-sectional \\
\hline Characteristics & - Study region: endemically occurring high fluoride \\
& - Investigated endpoint: IQ \\
\hline
\end{tabular}

\begin{tabular}{|l|l|l|}
\hline \multicolumn{1}{|c|}{ Strength } & \multicolumn{1}{|c|}{ Limitations } \\
\hline \begin{tabular}{|l|l|}
\hline \multicolumn{1}{|c|}{ Individual exposure assessment (water fluoride } \\
concentrations; urinary fluoride concentrations)
\end{tabular} & $\begin{array}{l}\text { Cross-sectional study design } \\
\text { Only a small percentage provided biological samples } \\
\text { Conducted in an endemic fluoride area but the impact by } \\
\text { other neurotoxicants (lead, mercury, arsenic) was not } \\
\text { considered } \\
\text { Incomplete set of covariates and sensitivity variables was } \\
\text { used (diet, oral hygiene, body mass index, and } \\
\text { socioeconomic status) } \\
\text { Urinary fluoride measures were not corrected for } \\
\text { dilution }\end{array}$ \\
\hline
\end{tabular}

Explanation: The study by Soto-Barreras et al. is a cross-sectional study which investigated $16 \mathrm{I}$ children in Chichuahua, Mexico, from 9 to 10 years of age. This study has the general limitations of the study type of cross-sectional studies already discussed above. However, the concentration of fluoride in drinking water (range: $0.79-1.48 \mathrm{mg} / \mathrm{L}$ ) and urine was analyzed individually but the urinary fluoride concentrations were not corrected for dilution by creatinine or specific gravity. The intellectual ability of children was evaluated through the Raven's Colored Progressive Matrices. Variables such as diet, oral hygiene, body mass index, and socioeconomic status were included. However, important factors known to influence children's IQ such as breastfeeding, low birth weight, and exposure to other neurotoxic chemicals were not analyzed. No relationship was found between intellectual ability and fluoride exposure variables such as dental fluorosis, levels of fluoride in drinking water and urine, and exposure dose. According to the authors the results suggest that fluoride exposure above $1.0 \mathrm{mg} / \mathrm{L}$ reduces the prevalence of dental caries, but no association was found with the intelligence of children. Thus, the study results are in agreement with the conclusions drawn by Guth et al. (2020) which do not support the presumption that fluoride should be assessed as a human developmental neurotoxicant at the current exposure levels in Europe. 
Till et al. 2020

\section{Key message}

Exposure to increasing levels of fluoride in tap water was associated with diminished non-verbal intellectual abilities; the effect was more pronounced among formula-fed children.

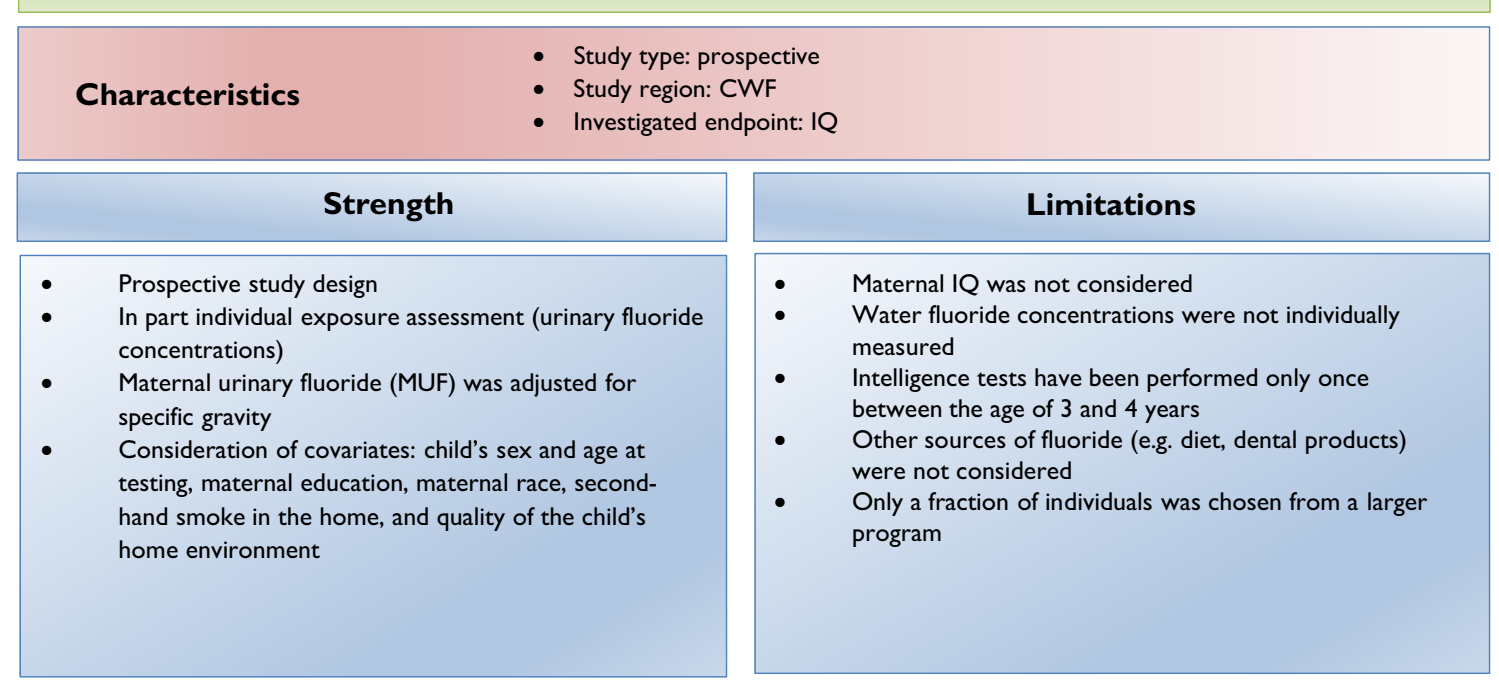

Explanation: The study of Till et al. was published online 2019 after the search period of Guth et al. (2020) and used the same MIREC cohort in Canada reported already by Green et al. (2019). 398 mother-child dyads who reported drinking tap water were examined. The water fluoride concentration was estimated using municipal water reports. Linear regression was used to analyze the association between fluoride exposure and IQ scores, measured by the Wechsler Primary and Preschool Scale of Intelligence-III at 3-4 years. It was examined whether feeding status (breast-fed (BF) versus formula-fed (FF)) modified the impact of water fluoride and if fluoride exposure during fetal development attenuated this effect. Covariate adjustment included child's sex and age at testing, maternal education (dichotomized as either a bachelor's degree or higher versus trade school diploma or lower), maternal race (white or other), second-hand smoke in the home (yes, no), and quality of the child's home environment (measured at time of testing using the Home Observation for Measurement of the Environment (HOME) - Revised Edition).

A $0.5 \mathrm{mg} / \mathrm{L}$ increase in water fluoride concentration was associated with a decrease of 4.4 Full Scale IQ (FSIQ) points $(95 \% \mathrm{Cl}:-8.34$, $-0.46, p=.03)$ in the FF group, but it was not significantly associated with FSIQ in the BF group (B=-1.34, 95\% Cl: $-5.04,2.38, p=.48)$. However, removing only two cases with extreme IQ scores from the models resulted in non-significant associations between water fluoride concentration and FSIQ in both groups. According to the authors the association between water fluoride concentration and FSIQ must be interpreted with caution, because the association became non-significant when the two outliers were removed. Controlling for fetal exposure by adding maternal urinary fluoride (MUF) to the model resulted in non-significant associations between water fluoride concentration and FSIQ in both the FF and BF groups.

The water fluoride concentration was significantly associated with lower Performance IQ (PIQ) in the $\mathrm{FF}(\mathrm{B}=-9.26,95 \% \mathrm{Cl}:-13.77$, $-4.76, p<.001)$ and the $B F$ groups $(B=-6.19,95 \% \mathrm{Cl}:-10.45,-1.94, p=.004)$. The association between water fluoride concentration and Performance IQ remained significant after controlling for fetal fluoride exposure among formula-fed. As mentioned by Till et al., breastmilk contains extremely low concentrations of fluoride $(0.005-0.01 \mathrm{mg} / \mathrm{L})$ due to the limited transfer of fluoride in plasma into breastmilk. Therefore, it seems unlikely that water fluoride concentration seems to play a role for fluoride exposure among exclusively breastfed children and that this could have an effect on IQ. Children in the breastfed group had higher FSIQ and Verbal IQ (VIQ) scores relative to the formula-fed group, regardless of fluoridation status. This is consistent with prior studies showing a positive effect of breastfeeding on cognition, amongst others the Broadbent et al. study (2015) which found that breastfeeding was associated with higher child IQ irrespective of residence in CWF or non-CWF areas. The authors recognize that higher education and income levels in the breastfed group (and so potentially higher IQ of the mothers) likely accounts for part of this association.

Overall, Till et al. present a well-conducted prospective cohort study based on the same cohort as the Green et al. study which we discussed in detail in our review (Guth et al., 2020). The authors suggested that the developing brain may also be adversely affected by fluoride exposure during infancy but also discussed the limitations of their study. Our review has pointed out some further limitations of the Green et al. study which also apply to the Till et al. study. A limitation of both studies is the lack of IQ data of the mothers. An additional limitation also applying to Green et al. is that the intelligence tests have been performed only once between the age of 3 and 4 years, but the exact age of the children at the time point of the test has not been considered in the statistical analysis. This may be problematic, because the IQ of children changes strongly between 3 and 4 years. 
Fig. 1 Correlation of maternal urinary fluoride concentration and full-scale IQ (FSIQ), reproduced from Green et al., 2019. Using this set of data, the authors concluded: "An increase from the 10th to 90th percentile of maternal urinary fluoride was associated with a 3.14 IQ decrement among boys." (Green et al. 2019). However, because of the relatively high variability of the IQ data, recently calculated benchmark doses of human neurotoxicity (Grandjean 2019) should be treated with caution

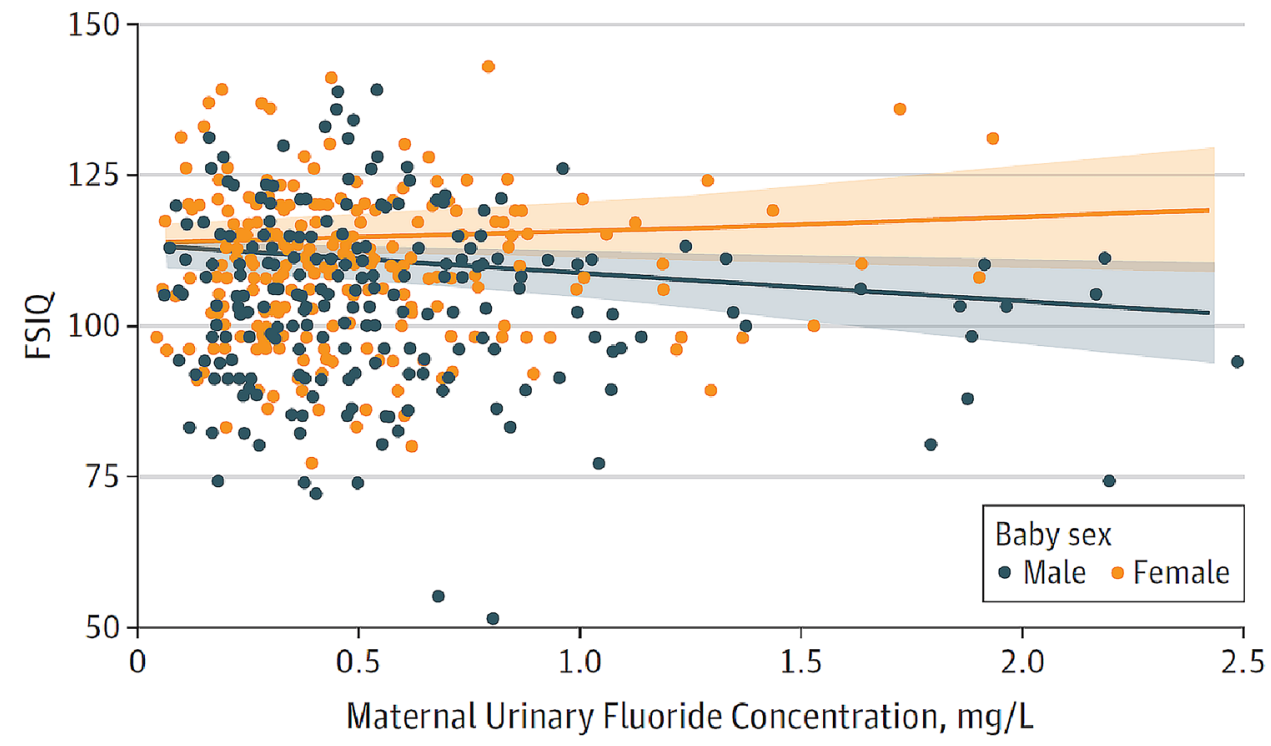

\section{Discrepancy between experimental and epidemiological evidence}

We observed a discrepancy between experimental and epidemiological evidence, which may be explained by deficiencies that were inherent to most of the current epidemiological studies, e.g. insufficient consideration of potential confounders. The majority of epidemiological studies which reported an association between lower measures of intelligence and high fluoride exposure was conducted in areas with endemically occurring high fluoride levels in ground and drinking water. In contrast, the experimental evidence suggests that current exposure to fluoride, even for individuals with relatively high fluoride intake, is clearly below levels that have led to adverse effects in vitro or in animals.

\section{Reasons why it is problematic to calculate bench mark doses for humans (BMD)}

A main criticism of our review was that we made "no attempt to calculate the threshold for fluoride neurotoxicity using the standard benchmark dose method.'...'Grandjean singled out three prospective studies, two in 2017 from Mexico by Valdez Jiménez et al. and Bashash et al. and one in 2018 from Canada by Green et al., ... to estimate the tentative benchmark dose (BMD) values.' (Spittle 2020).

Grandjean used the regression coefficients and their standard deviations as provided in the published reports to estimate tentative BMD values. A BMDL of about $0.2 \mathrm{mg} / \mathrm{L}$ or below was suggested (Grandjean 2019), which was similar to the result calculated from a previous study (Xiang et al. 2003) by Hirzy et al. (Hirzy et al. 2016) (for a brief discussion of studies calculating BMDs for humans see
Box 6). It should be considered that even without fluoridation, the fluoride concentration in drinking water in Europe often ranges around $0.5 \mathrm{mg} / \mathrm{L}$ and is therefore higher than the BMDL of $0.2 \mathrm{mg} / \mathrm{L}$ derived by Grandjean et al. (2019). It was concluded that the benchmark dose of fluoride neurotoxicity is clearly below commonly occurring fluoride exposure levels.

We did not follow this approach to calculate the BMD, because the results of such calculations would be questionable due to the inadequate quality of the available input data. It remains unclear why two studies (Bashash et al. 2017; Green et al. 2019) were finally selected to calculate the BMD (Grandjean 2019); whereas, others with a negative result (Broadbent et al. 2015) were omitted. The studies by Valdez Jimenez et al. (2017) and Bashash et al. (2017) have limitations, such as the lack of control of the influence of other neurotoxicants and small sample size (Box 4). Green et al. (2019) openly discussed the limitations of their own study directly in their publication, which are briefly summarized in Box 5. The difficulty to 'calculate the threshold of fluoride neurotoxicity' (Spittle 2020) is illustrated using a scatter plot of IQ (FSIQ) versus maternal urinary fluoride, where each dot represents the IQ of a child (Fig. 1; reproduced Fig. 3A from (Green et al. 2019)). The trend line of IQ of the girls slightly increased with higher maternal urinary fluoride, although this effect was not statistically significant. In contrast, a significantly lower IQ was observed for boys, which depended on the two individuals with the highest urinary fluoride (Fig. 1). This difference led to the suggestion that there may be a sex difference in response to fluoride (Grandjean 2019). However, considering the high overall variability of IQ among the children in the study, this interpretation should be done with caution. Rather, further studies are required before such conclusions can be drawn. 
Box 5 Two prospective studies already considered in Guth et al (2020)

\section{Broadbent et al. 2015}

\section{Key message}

No clear differences in IQ because of fluoride exposure via community water fluoridation (CWF) were noted. The findings of the study do not support the assertion that fluoride in the context of CWF programs is neurotoxic.

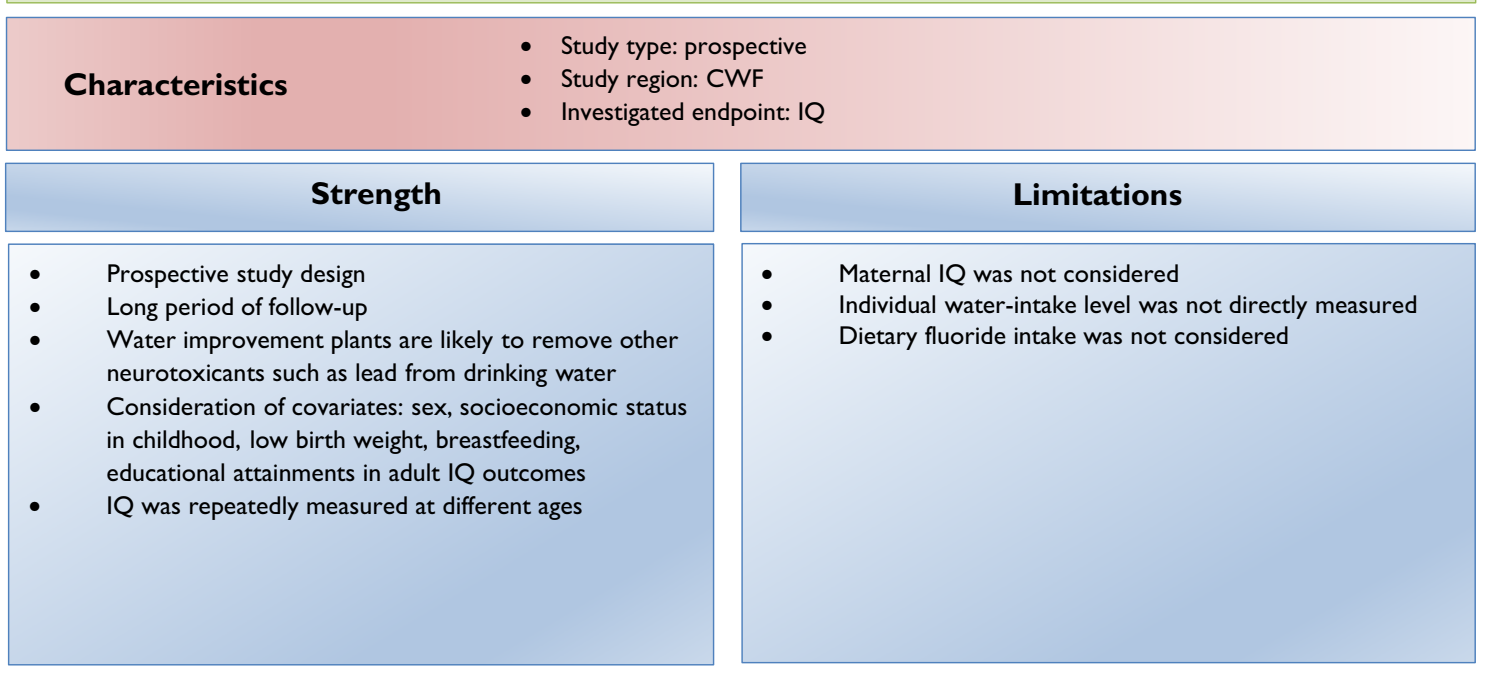

Explanation: We analyzed this study in detail in our review article (Guth et al. 2020). The study was performed with a general population sample of 1.037 children born in Dunedin, New Zealand. The participants were followed for 38 years and their fluoride intake via drinking water (residence in a CWF area versus non-CWF area; 0.7-1.0 mg fluoride/L vs. $0.0-0.3 \mathrm{mg}$ fluoride/L), fluoride dentifrice, and/or $0.5 \mathrm{mg}$ fluoride tablets in early life (prior to age 5 years) was deduced. IQ was assessed repeatedly between ages 7 and 13 years and at age 38 years. It was reported that no statistically significant differences in IQ due to fluoride exposure were observed also following adjustment for potential confounding variables, including sex, socioeconomic status, breastfeeding, and birth weight (as well as educational attainment for adult IQ outcomes).

It was one of two available studies with a suitable study design (prospective cohort study) conducted in a non-endemic CWF area that appropriately considered confounding factors. However, we also identified some limitations already described in our review. For example, parental IQ is a strong confounder but the IQ data of the mothers were lacking. Furthermore, a limitation of the study is the fact that individual water-intake level was not directly measured and dietary fluoride was not considered.

The study by Broadbent et al. was not only classified in our review as a high quality study but was also classified as such by other bodies e.g. the Health Board of Ireland (Sutton et al. 2015) or the National Health and Medical Research Council (NHMRC) of the Australian Government (NHMRC 2017). These bodies stated in agreement with our review that the study by Broadbent et al. is a high quality prospective cohort study, with a low risk of bias (NHMCR 2017). Moreover, this study is one of the very few prospective cohort studies conducted in a CWF area and its design is appropriate for inferring causality (Sutton et al. 2015).

The Broadbent study has been critiqued (e.g. by Spittle 2020) on the grounds that the estimated difference in the exposure to fluoride, from drinking water, food, toothpaste, beverages, and fluoride supplements, of less than $0.2 \mathrm{mg} /$ day, between the CWF group and the non-CWF group was so small that it was unlikely to lead to a detectable difference in IQ (Hirzy et al. 2016 was cited).

Broadbent et al. reported residence in an area with or without CWF (0.7--1.0 ppm and 0.0--0.3 ppm fluoride, respectively) coded from residential address data (Broadbent et a. 2015). Hirzy et al. (2016) estimated a daily intake of I.36 and I.19 mg/day, respectively, for the CWF and non-CWF group. This was based on several assumptions, e.g. that a relatively high proportion of the overall exposure results from dietary sources or supplements. Osmunson et al. also calculated total fluoride intake for the CWF and non-CWF Dunedin Cohort participants using publicly available data (Osmunson et al. 2016) and estimated that lifetime CWF children had a mean total fluoride intake of $0.7 \mathrm{mg} /$ day while non-CWF children averaged $0.5 \mathrm{mg} /$ day. Broadbent et al responded to this estimation that it is similar to their own estimate of an average difference of a total daily fluoride intake of $0.3 \mathrm{mg} /$ day through the first five years of life between study members from CWF versus non-CWF areas. According to Broadbent et al. (2016) these differences are consistent with the literature. Other researchers have estimated that the increase in fluoride intake among children aged one to three years attributable to CWF is 0.2 milligrams per day (Rojas-Sanchez et al. 1999) or 0.3 milligrams per day (Cressey et al. 2010).

Even when considering that the difference between fluoride exposure in CWF and non-CWF areas is small, the results of the Broadbent study showed that living in a CWF or non-CWF did not have a significant effect on the IQ of children suggesting that fluoride drinking water concentrations in the range of CWF do not represent a health concern. It is reasonable that this difference is much smaller in CWF areas than the difference occurring in endemic fluoride areas with a very high fluoride concentration of drinking water. 
Green et al. 2019

\section{Key message}

In this study maternal exposure to higher levels of fluoride during pregnancy was associated with lower IQ scores in children aged 3 to 4 years.

\section{Characteristics \\ - Study type: prospective \\ - Study region: CWF \\ - Investigated endpoint: IQ}

\section{Strength}

- $\quad$ Prospective study design

- In part individual exposure assessment (urinary fluoride concentrations)

- Water improvement plants are likely to remove other neurotoxicants such as lead from drinking water

- Maternal urinary fluoride (MUF) was adjusted for specific gravity and creatinine

- Relevant covariates were considered: city, socioeconomic status, maternal education, race/ethnicity, prenatal secondhand smoke exposure

\section{Limitations}

Maternal IQ was not considered

- Intelligence tests have been performed only once between the age of 3 and 4 years

- $\quad$ Covariates not considered: breastfeeding, low birth weight, alcohol consumption, exact age of children at time point of testing

- Assessment of children's postnatal fluoride exposure via, e.g., diet, fluoride dentifrice, and/or fluoride tablets, was not included

- $\quad$ Only a fraction of the individuals from a larger program was chosen for the study

Explanation: We analyzed this study in detail in our review article (Guth et al. 2020). It was one of two available studies with a suitable study design (prospective cohort study) conducted in a non-endemic CWF area that also appropriately considered confounding factors (even though there are still some limitations, see below).

A limitation of the study is the lack of IQ data of the mothers, because parental IQ is a strong confounder. Moreover, it cannot be excluded that the 'outcome' (intelligence) influenced the 'input' (fluoride exposure). This is an important possible confounder which is frequently not considered. It is possible that more intelligent mothers read or inform themselves more about possible health hazards of children and therefore avoid fluoride exposure. In this case high maternal intelligence would be causally linked to lower fluoride exposure rather than high fluoride exposure causing lower intelligence of children. An additional limitation is that the intelligence tests have been performed only once between the age of 3 and 4 years but the exact age of the children at the time point of the test has not been considered in the statistical analysis. This may be problematic, because the IQ of children changes strongly between 3 and 4 years. Moreover, only 610 of 2001 pregnant women from the MIREC program were considered and information on maternal urinary fluoride was missing in a relatively high fraction of the mothers of children of whom IQ was determined. This may represent a possible source of bias.

Green et al. (2019) considered some of the relevant confounders (city, socioeconomic status, maternal education, race/ethnicity, prenatal secondhand smoke exposure) but did not adjust for others (breastfeeding, low birth weight, alcohol consumption and further dietary factors, other sources of fluoride exposure, exact age of children at time point of testing). Furthermore, assessment of children's postnatal fluoride exposure via, e.g., diet, fluoride dentifrice, and/or fluoride tablets, was not included. 
Box 6 Brief discussion of studies calculating benchmark doses (BMD) for humans

Hirzy et al. (2016)

Hirzy et al. (2016) used the benchmark approach to generate benchmark results from the study by Xiang et al. (2003). The authors discussed the limitations of their study and stated that the calculated reference dose is based on a limited amount of quantitative data, most of which is of ecological design. Furthermore, they were unable to find any data on human intellectual performance as a function of fluoride exposure in the USA. Nor were there studies, other than those by the Xiang et al. research group, which provided any useful dose-response information. In estimating RfD values, Hirzy et al. used mean water consumption rates, except as noted, and mean IQ measurements that were derived from different testing methods, recognizing the limitations of these uses and those inherent in ecological studies generally. The authors stated that the data they used for the food component in estimating total fluoride intake were mean values from one study that were not accompanied by standard deviations. They were, however, somewhat higher than the values for children's food fluoride exposures in the USA indicating a conservative approach. Overall, Hirzy et al. acknowledged that it would have been useful to have a more robust data set on which to base the risk analysis, but in their opinion waiting for more such data that are unlikely to be developed in the near future did not seem reasonable. Because of these limitations, already discussed by the authors, we recommend not to use the data for calculation of a reference dose and exposure limit. As concluded in our review article, further research is needed for a comprehensive risk assessment, for example, high-quality prospective epidemiological studies that adequately control for any confounding factors.

\section{Grandjean (2019)}

Grandjean (2019) focused on human epidemiological studies published subsequent to a meta-analysis from 2012 (Choi et al. 2012), and did not include evidence from animal and in vitro experiments. The authors reported that fourteen recently published cross-sectional studies from endemic areas with naturally occurring high fluoride concentrations in groundwater supported the findings of cognitive deficits in children with elevated fluoride exposures reported in 2012. Furthermore, Grandjean analyzed three prospective studies from Mexico and Canada with individual exposure data (Valdez Jiménez et al. 2017; Bashash et al. 2017 and Green et al. 2019) and reported that based on these studies early-life exposures were negatively associated with children's performance in cognitive tests. According to the conclusions drawn by Grandjean, neurotoxicity appeared to be dose-dependent, and he conducted a tentative benchmark dose calculation, which suggests that safe exposures are likely to be below currently accepted or recommended fluoride concentrations in drinking water. As a result, he concluded that the recent epidemiological studies support the notion that elevated fluoride intake during early development can result in IQ deficits that may be considerable. Considering the high overall variability of $I Q$, this interpretation should be treated with caution. Rather, further studies are required before such conclusions can be drawn.

\section{Conclusion}

There are varying opinions on the health effects of high fluoride exposure. Our recent assessment was based on evidence from animal, in vitro and epidemiological studies focusing on exposure scenarios relevant for the population in Europe. Others included epidemiological evidence from endemic areas into their assessment. Moreover, we critically discussed the insufficient consideration of confounding factors and deficiencies of study design and statistical evaluation in available epidemiological studies. Thus, the differences in considered study populations and different standards in evaluating the quality of epidemiological studies may at least in part explain the different assessments. Also, considering the additional studies which did not meet the inclusion criteria of our first review article (see Box 4), we still arrive at the same conclusions: the available epidemiological evidence does not provide sufficient arguments to raise concerns with regard to CWF in the range of $0.7-1.0 \mathrm{mg} / \mathrm{L}$, nor does it justify that fluoride should be categorized as a human developmental neurotoxicant, signifying that it is similarly problematic as lead or methylmercury at current exposure levels. Of course, the conclusions may have to be reconsidered if new comprehensive findings from epidemiological or animal studies are presented.

\section{Final recommendations}

Calculation of a threshold for human fluoride neurotoxicity based on selected epidemiological studies may be problematic since the available data are not considered to be sufficient to perform a dose response assessment. For risk evaluation, it is important to consider all available data, including animal experiments and in vitro studies. Further animal studies and prospective epidemiological studies would be 
helpful, but should avoid the limitations of previous investigations as summarized in Box 1, Box 2 and described by Guth et al. (2020).

Acknowledgements This study was an activity of the Senate Commission on Food Safety (SKLM) of the German Research Foundation (DFG). The authors wish to thank the DFG for their continuous support of the SKLM Commission.

Funding Open Access funding enabled and organized by Projekt DEAL.

Open Access This article is licensed under a Creative Commons Attribution 4.0 International License, which permits use, sharing, adaptation, distribution and reproduction in any medium or format, as long as you give appropriate credit to the original author(s) and the source, provide a link to the Creative Commons licence, and indicate if changes were made. The images or other third party material in this article are included in the article's Creative Commons licence, unless indicated otherwise in a credit line to the material. If material is not included in the article's Creative Commons licence and your intended use is not permitted by statutory regulation or exceeds the permitted use, you will need to obtain permission directly from the copyright holder. To view a copy of this licence, visit http://creativecommons.org/licenses/by/4.0/.

\section{References}

Aggeborn L, Oehman M (2017) The effects of fluoride in drinking water. Uppsala: Institute for Evaluation of Labour Market and Education Policy, p. 1-83. https://www.ifau.se/globalassets/pdf/ se/2017/wp2017-20-the-effects-of-fluoride-in-the-drinking-water. pdf

Aggeborn L, Oehman M (2021) The effects of fluoride in drinking water. Journal of Political Economy 129(2):465-491. https://www. journals.uchicago.edu/doi/pdf/doi.org/10.1086/711915

Bashash M, Thomas D, Hu H et al (2017) Prenatal fluoride exposure and cognitive outcomes in children at 4 and 6-12 years of age in Mexico. Environ Health Perspect 125(9):097017. https://doi.org/ 10.1289/EHP655

Broadbent JM, Thomson WM, Ramrakha S et al (2015) Community water fluoridation and intelligence: prospective study in New Zealand. Am J Public Health 105(1):72-76. https://doi.org/10.2105/ AJPH.2013.301857

Broadbent JM, Thomson WM, Moffitt TE, Poulton RBroadbent, et al (2016) Respond. Am J Public Health 106(2):213-214. https://doi. org/10.2105/AJPH.2015.303013

Choi AL, Sun G, Zhang Y, Grandjean P (2012) Developmental fluoride neurotoxicity: a systematic review and meta-analysis. Environ Health Perspect 120(10):1362-1368. https://doi.org/10.1289/ehp. 1104912

Claus Henn B, Austin C, Coull BA et al (2018) Uncovering neurodevelopmental windows of susceptibility to manganese exposure using dentine microspatial analyses. Environ Res 161:588-598. https:// doi.org/10.1016/j.envres.2017.12.003

Collins TF, Sprando RL, Shackelford ME et al (1995) Developmental toxicity of sodium fluoride in rats. Food Chem Toxicol 33(11):951-960

Collins TF, Sprando RL, Black TN et al (2001) Developmental toxicity of sodium fluoride measured during multiple generations. Food Chem Toxicol 39(8):867-876
Cressey P, Gaw S, Love J (2010) Estimated dietary fluoride intake for New Zealanders. J Public Health Dent 70(4):327-336. https://doi. org/10.1111/j.1752-7325.2010.00192.x

Dunipace AJ, Brizendine EJ, Zhang W et al (1995) Effect of aging on animal response to chronic fluoride exposure. J Dent Res 74(1):358-368

EFSA (2013) Panel on dietetic products, nutrition; scientific opinion on dietary reference values for fluoride. EFSA J 11(8):3332

Feldman V (2014) Neurodevelopmental toxicity: still more questions than answers. Lancet Neurol 13(7):645-646. https://doi.org/10. 1016/S1474-4422(14)70115-2

Gelinas J, Allukian M Jr (2014) Neurodevelopmental toxicity: still more questions than answers. Lancet Neurol 13(7):647-648. https://doi.org/10.1016/S1474-4422(14)70119-X

Godoy P, Hewitt NJ, Albrecht U et al (2013) Recent advances in 2D and $3 \mathrm{D}$ in vitro systems using primary hepatocytes, alternative hepatocyte sources and non-parenchymal liver cells and their use in investigating mechanisms of hepatotoxicity, cell signaling and ADME. Arch Toxicol 87(8):1315-1530. https://doi.org/10.1007/ s00204-013-1078-5

Grandjean P (2019) Developmental fluoride neurotoxicity: an updated review. Environ Health 18(1):110. https://doi.org/10.1186/ s12940-019-0551-x

Grandjean P, Landrigan PJ (2014) Neurobehavioural effects of developmental toxicity. Lancet Neurol 13(3):330-338. https://doi.org/ 10.1016/S1474-4422(13)70278-3

Green R, Lanphear B, Hornung R et al (2019) Association between maternal fluoride exposure during pregnancy and IQ scores in offspring in Canada. JAMA Pediatr 173(10):940-948. https://doi. org/10.1001/jamapediatrics.2019.1729

Guth S, Huser S, Roth A et al (2020) Toxicity of fluoride: critical evaluation of evidence for human developmental neurotoxicity in epidemiological studies, animal experiments and in vitro analyses. Arch Toxicol 94(5):1375-1415. https://doi.org/10.1007/ s00204-020-02725-2

Heindel JJ, Bates HK, Price CJ, Marr MC, Myers CB, Schwetz BA (1996) Developmental toxicity evaluation of sodium fluoride administered to rats and rabbits in drinking water. Fundam Appl Toxicol 30(2):162-177

Hirzy JW, Connett P, Xiang QY, Spittle BJ, Kennedy DC (2016) Developmental neurotoxicity of fluoride: a quantitative risk analysis towards establishing a safe daily dose of fluoride for children. Fluoride 49(4):379-400

Kupsco A, Sanchez-Guerra M, Amarasiriwardena C et al (2019) Prenatal manganese and cord blood mitochondrial DNA copy number: Effect modification by maternal anemic status. Environ Int 126:484-493. https://doi.org/10.1016/j.envint.2019.02.029

Leist M (2017) New animal-free concepts and test methods for developmental toxicity and peripheral neurotoxicity. Altern Lab Anim 45(5):253-260. https://doi.org/10.1177/026119291704500505

Lewis RC, Meeker JD, Basu N et al (2018) Urinary metal concentrations among mothers and children in a Mexico City birth cohort study. Int J Hyg Environ Health 221(4):609-615. https://doi.org/ 10.1016/j.ijheh.2018.04.005

McPherson CA, Zhang G, Gilliam R et al (2018) An Evaluation of Neurotoxicity Following Fluoride Exposure from Gestational Through Adult Ages in Long-Evans Hooded Rats. Neurotox Res 34(4):781-798. https://doi.org/10.1007/s12640-018-9870-x

Mullin AM, Amarasiriwardena C, Cantoral-Preciado A et al (2019) Maternal blood arsenic levels and associations with birth weightfor-gestational age. Environ Res 177:108603. https://doi.org/10. 1016/j.envres.2019.108603

NHMRC (2017) National Health and Medical Research Council. Information paper - Water fluoridation: dental and other human health outcomes, report prepared by the Clinical Trials Centre at 
University of Sydney, NHMRC; Canberra. https://www.nhmrc. gov.au/sites/default/files/documents/reports/fluoridation-infopaper.pdf.

NRC (2006) National Research Council; Fluoride in Drinking Water: A Scientific Review of EPA's Standards. Committee on Fluoride in Drinking Water. Board on Environmental Studies and Toxicology. Division on Earth and Life Studies. The National Academy Press, Washington DC, USA.https://doi.org/1017226/11571

NTP (2016) National Toxicology Program. Systematic literature review on the effects of fluoride on learning and memory in animal studies. NTP Research Report 1. Research Triangle Park, NC: National Toxicology Program.

Orta-Garcia S, Perez-Vazquez F, Gonzalez-Vega C, Varela-Silva JA, Hernandez-Gonzalez L, Perez-Maldonado I (2014) Concentrations of persistent organic pollutants (POPs) in human blood samples from Mexico City, Mexico. Sci Total Environ 472:496-501. https://doi.org/10.1016/j.scitotenv.2013.11.059

Osmunson B, Limeback H, Neurath C (2016) Study Incapable Of Detecting IQ Loss From Fluoride. Am J Public Health 106(2):212-213. https://doi.org/10.2105/AJPH.2015.302918

Pulungan ZSA, Sofro ZM, Partadiredja G (2018) Sodium fluoride does not affect the working memory and number of pyramidal cells in rat medial prefrontal cortex. Anat Sci Int 93(1):128-138. https:// doi.org/10.1007/s12565-016-0384-4

Rojas-Sanchez F, Kelly SA, Drake KM, Eckert GJ, Stookey GK, Dunipace AJ (1999) Fluoride intake from foods, beverages and dentifrice by young children in communities with negligibly and optimally fluoridated water: a pilot study. Community Dent Oral Epidemiol 27(4):288-297. https://doi.org/10.1111/j.1600-0528. 1998.tb02023.x

Rugg-Gunn AJ, Villa AE, Buzalaf MR (2011) Contemporary biological markers of exposure to fluoride. Monogr Oral Sci 22(37-5): 1

Soto-Barreras U, Escalante-Villalobos KY, Holguín-Loya B et al (2019) Effect of fluoride in drinking water on dental caries and IQ in children. Fluoride 52(3):474-482

Spittle B et al (2020) Reviews of developmental neurotoxicity by Grandjean and Guth et al. Fluoride 53(2):204-219
Sun H, Como PG, Downey LC, Murphy D, Ariagno RL, Rodriguez W (2015) Infant formula and neurocognitive outcomes: impact of study end-point selection. J Perinatol 35(10):867-874. https://doi. org/10.1038/jp.2015.87

Sutton M, Kiersey R, Farragher L, Long J (2015) Health effects of water fluoridation. An evidence review. Health Research Board, Ireland. https://www.hrb.ie/fileadmin/publications_files/Health_ Effects_of_Water_Fluoridation.pdf.

Till C, Green R, Flora D et al (2020) Fluoride exposure from infant formula and child IQ in a Canadian birth cohort. Environ Int 134:105315. https://doi.org/10.1016/j.envint.2019.105315

Valdez Jimenez L, Lopez Guzman OD, Cervantes Flores M et al (2017) In utero exposure to fluoride and cognitive development delay in infants. Neurotoxicology 59:65-70. https://doi.org/10.1016/j. neuro.2016.12.011

Watkins DJ, Fortenberry GZ, Sanchez BN et al (2016) Urinary 3-phenoxybenzoic acid (3-PBA) levels among pregnant women in Mexico City: Distribution and relationships with child neurodevelopment. Environ Res 147:307-313. https://doi.org/10.1016/j. envres.2016.02.025

Watkins DJ, Sanchez BN, Tellez-Rojo MM et al (2017) Phthalate and bisphenol A exposure during in utero windows of susceptibility in relation to reproductive hormones and pubertal development in girls. Environ Res 159:143-151. https://doi.org/10.1016/j.envres. 2017.07.051

Xiang Q, Liang Y, Chen L, et al. (2003) Effect of fluoride in drinking water on children's intelligence. Fluoride 36(2):84-94. Erratum in Fluoride 2004;37(4):320.

Publisher's Note Springer Nature remains neutral with regard to jurisdictional claims in published maps and institutional affiliations.

\title{
Authors and Affiliations
}

\author{
Sabine Guth ${ }^{1}\left[\right.$ - Stephanie Hüser ${ }^{1} \cdot$ Angelika Roth $^{1} \cdot$ Gisela Degen $^{1} \cdot$ Patrick Diel $^{2} \cdot$ Karolina Edlund $^{1}$. \\ Gerhard Eisenbrand ${ }^{3} \cdot$ Karl-Heinz Engel $^{4} \cdot$ Bernd Epe $^{5}$. Tilman Grune ${ }^{6} \cdot$ Volker Heinz $^{7} \cdot$ Thomas Henle $^{8}$. \\ Hans-Ulrich Humpf ${ }^{9} \cdot$ Henry Jäger $^{10} \cdot$ Hans-Georg Joost ${ }^{11} \cdot$ Sabine E. Kulling ${ }^{12} \cdot$ Alfonso Lampen $^{13} \cdot$ Angela Mally $^{14}$. \\ Rosemarie Marchan ${ }^{1}$. Doris Marko ${ }^{15}$. Eva Mühle ${ }^{1}$. Michael A. Nitsche ${ }^{16,17}$. Elke Röhrdanz ${ }^{18}$. Richard Stadler ${ }^{19}$. \\ Christoph van Thriel ${ }^{1} \cdot$ Stefan Vieths ${ }^{20} \cdot$ Rudi F. Vogel $^{21} \cdot$ Edmund Wascher $^{22} \cdot$ Carsten Watzl $^{23} \cdot$ Ute Nöthlings $^{24}$. \\ Jan G. Hengstler ${ }^{1}$
}

1 Department of Toxicology, Leibniz Research Centre for Working Environment and Human Factors (IfADo), Dortmund, Germany

2 Department of Molecular and Cellular Sports Medicine, Institute of Cardiovascular Research and Sports Medicine, German Sport University Cologne, Cologne, Germany

3 Kühler Grund 48/1, 69126 Heidelberg, Germany
4 Department of General Food Technology, School of Life Sciences, TU Munich, Freising, Germany

5 Institute of Pharmacy and Biochemistry, University of Mainz, Mainz, Germany

6 Department of Molecular Toxicology, German Institute of Human Nutrition (DIfE), Nuthetal, Germany 
7 German Institute of Food Technologies (DIL), Quakenbrück, Germany

8 Department of Food Chemistry, TU Dresden, Dresden, Germany

9 Institute of Food Chemistry, Westfälische Wilhelms-Universität Münster, Münster, Germany

10 Institute of Food Technology, University of Natural Resources and Life Sciences (BOKU), Vienna, Austria

11 Department of Experimental Diabetology, German Institute of Human Nutrition (DIfE), Nuthetal, Germany

12 Department of Safety and Quality of Fruit and Vegetables, Max Rubner-Institut, Federal Research Institute of Nutrition and Food, Karlsruhe, Germany

13 Department of Food Safety, Bundesinstitut für Risikobewertung (BfR), Berlin, Germany

14 Department of Toxicology, University of Würzburg, Würzburg, Germany

15 Department of Food Chemistry and Toxicology, Faculty of Chemistry, University of Vienna, Vienna, Austria

16 Department of Psychology and Neurosciences, Leibniz Research Centre for Working Environment and Human Factors (IfADo), Dortmund, Germany

17 Department of Neurology, University Medical Hospital Bergmannsheil, Ruhr-University, Bochum, Germany
18 Department of Experimental Pharmacology and Toxicology, Federal Institute for Drugs and Medical Devices (BfArM), Bonn, Germany

19 Institute of Food Safety and Analytic Sciences, Nestlé Research Centre, Lausanne, Switzerland

20 Paul-Ehrlich-Institut, Langen, Germany

21 Lehrstuhl für Technische Mikrobiologie, TU Munich, Freising, Germany

22 Department of Ergonomics, Leibniz Research Centre for Working Environment and Human Factors (IfADo), Dortmund, Germany

23 Department of Immunology, Leibniz Research Centre for Working Environment and Human Factors (IfADo), Dortmund, Germany

24 Department of Nutrition and Food Sciences, Nutritional Epidemiology,

Rheinische Friedrich-Wilhelms University Bonn, Bonn, Germany 